\title{
Electron momentum and energy relaxation rates in GaN and AIN in the high-field transport regime
}

\author{
C. Bulutay* \\ Department of Physics, Bilkent University, 06533 Bilkent, Ankara, Turkey \\ B. K. Ridley ${ }^{\dagger}$ \\ Department of Electronic Systems Engineering, University of Essex, Colchester C04 3SQ, United Kingdom \\ N. A. Zakhleniuk \\ Bookham Technology, Caswell, Towcester, Northants NN12 8EQ, United Kingdom
}

(Received 14 April 2003; published 26 September 2003)

\begin{abstract}
Momentum and energy relaxation characteristics of electrons in the conduction band of GaN and AlN are investigated using two different theoretical approaches corresponding to two high electric-field regimes, one up to 1-2 $\mathrm{MV} / \mathrm{cm}$ values for incoherent dynamics, and the other at even higher fields for coherent dynamics where semiballistic and ballistic processes become important. For the former, ensemble Monte Carlo technique is utilized to evaluate these rates as a function of electron energy up to an electric-field value of $1 \mathrm{MV} / \mathrm{cm}(2$ $\mathrm{MV} / \mathrm{cm}$ ) for $\mathrm{GaN}(\mathrm{AlN})$. Momentum and energy relaxation rates within this incoherent transport regime in the presence of all standard scattering mechanisms are computed as well as the average drift velocity as a function of the applied field. Major scattering mechanisms are identified as polar optical phonon (POP) scattering and the optical deformation potential (ODP) scattering. Roughly, up to fields where the steady-state electron velocity attains its peak value, the POP mechanism dominates, whereas at higher fields ODP mechanism takes over. Next, aiming to characterize coherent dynamics, the total out-scattering rate from a quantum state (chosen along a high-symmetry direction) due to these two scattering mechanisms are then computed using a firstprinciples full-band approach. In the case of POP scattering, momentum relaxation rate differs from the total out-scattering rate from that state; close to the conduction-band minimum, momentum relaxation rate is significantly lower than the scattering rate because of forward-scattering character of the intravalley POP emission. However, close to the zone boundary the difference between these two rates diminishes due to isotropic nature of intervalley scatterings. Finally, a simple estimate for the velocity-field behavior in the coherent transport regime is attempted, displaying a negative differential mobility due to the negative band effective mass along the electric-field direction.
\end{abstract}

DOI: 10.1103/PhysRevB.68.115205

PACS number(s): 72.10.-d, 72.15.Lh, 72.20.Ht, 72.80.Ey

\section{INTRODUCTION}

Gallium nitride and aluminum nitride belong to wide band-gap materials having very desirable properties for high power applications. These two semiconductors form the basis of technologically important devices such as GaN/AlGaN high electron mobility transistors (HEMT's) [Refs. 1,2] and AlGaN solar-blind photodiodes. ${ }^{3}$ Being under attention for over a decade, the high-field phenomena close to breakdown fields (about a few $\mathrm{MV} / \mathrm{cm}$ ) in group III-nitride semiconductors still needs further scrutiny. Due to strong ionicity of the III-nitride bonds, polar optical phonons (POP) form the main scattering channel for the energetic electrons, as has been confirmed experimentally by several groups studying hotelectron energy and momentum relaxation in bulk, ${ }^{4}$ as well as in two-dimensional structures. ${ }^{5}$ However, the electric-field values in these experiments were still much lower than the prebreakdown regime ${ }^{6}$ which remains to be investigated. Another effective mechanism at higher energies is the optical deformation potential (ODP) scattering. ${ }^{7,8}$ The interplay between these two mechanisms governs the nature of carrier dynamics, and in particular explains the disparities among the carrier scattering times and the energy and momentum relaxation times.
Our previous analysis of the full-band electron-scattering rate due to POP emission indicated extremely efficient scattering for both $\mathrm{GaN}$ and $\mathrm{AlN} .^{9-11}$ A particular nature of the polar scattering is the fact that it favors small momentum exchanges. ${ }^{7,8}$ Therefore, the momentum relaxation rate should be significantly less than the scattering rate, which implies that under a high electric field even though an electron makes several collisions on its way to the zone boundary, its progress is not as impeded as the scattering rate would suggest. This difference between the momentum relaxation time and the scattering time is of crucial importance for assessing the feasibility of both the full-band electron dynamics in bulk semiconductors, Krömer-Esaki-Tsu regime, ${ }^{12,13}$ and the overshoot regime in GaN-based HEMT's with the gate length of a few tens of nanometers. ${ }^{2}$ In both of these cases the character of the electron motion in the momentum space is rather intermediate between the diffusionlike and the ballisticlike motion, which are characterized by different characteristic times, the momentum relaxation time, and the scattering time. These are followed by a slower, dissipative process, described by the energy relaxation time on the path to carrier thermalization. The slow rate of the electron energy dissipation is mainly due to smallness of the dissipation factor, $\delta=\Delta E / E \approx \hbar \omega_{0} / E$, at high energies $E \gg \hbar \omega_{0}$ ( $\hbar \omega_{0}$ is the optical phonon energy), where the en- 
ergy loss per collision $\Delta E$ is small in comparison with $E$, i.e., $\delta \ll 1$.

Aim of this work is to resolve the importance of competing scattering mechanisms and present a comprehensive quantitative account of the scattering, momentum and energy relaxation rates corresponding to these mechanisms for $\mathrm{GaN}$ and AlN. To avoid fundamental simplifications that will have adverse quantitative consequences, we start with the ensemble Monte Carlo (EMC) approach; Sec. II combines the details about the method and the results on momentum and energy relaxation rates. This analysis enables us to identify the dominant scattering mechanisms with respect to an applied electric field. Within the EMC framework, we can describe quantitatively ensemble average of any transportrelated property which is well suited for the incoherent carrier dynamics. A complementary approach suited for ballistic and semiballistic regimes is to investigate these scattering and relaxation rates at each quantum state $\mathbf{k}$, assuming an electron to be promoted to this state by a coherent optical or electronic excitation. Hence, we supplement the EMC analysis with a full-band, first-principles computation of these total out-scattering rates from a quantum state (chosen along a high-symmetry direction) due to each of these two scattering mechanisms. Section III gives a brief account of this computational methodology, followed by results and a discussion of the Krömer-Esaki-Tsu negative differential mobility; finally, our conclusions are given in Sec. IV.

\section{ENSEMBLE MONTE CARLO ANALYSIS}

For the high-field transport phenomena EMC technique is currently the most reliable choice, free from major simplifications; for an up to date account see, for instance, Ref. 14. We include the following scattering mechanisms in our EMC treatment: acoustic and optical deformation potential, polar optical phonon, ionized impurity, and the impact ionization scatterings. On the other hand, the piezoacoustic, neutral impurity, and dislocation scatterings are not included as they become significant at low temperatures and fields. ${ }^{78}$ Our EMC simulations are based on a temperature of $300 \mathrm{~K}$ with $10^{17} \mathrm{~cm}^{-3}$ electrons in the conduction band. Furthermore, we screen the POP and ionized impurity potentials using random-phase-approximation-based dielectric function ${ }^{15}$ and incorporate the state occupancy effects through the LugliFerry approach. ${ }^{16}$ In the case of sufficiently high excitation densities, it has been found that the distribution function of phonons is driven substantially out of equilibrium and that this "hot-phonon effect" may drastically reduce the cooling process. ${ }^{17}$ However, we do not consider such hot-phonon effects, even though they can be treated within the EMC framework. ${ }^{18}$

We extract the necessary band-edge energy, effective mass, and nonparabolicity parameters of all valleys (located at $\Gamma_{1}, U_{3, \min }, K, M$, and $\Gamma_{3}$ points) in the lowest two conduction bands from our empirical pseudopotential band structure for GaN (Ref. 9) and AlN; ${ }^{11}$ we refer to Ref. 19 for a full listing of these parameters as well as for our modeling of the impact ionization scattering which is however not so significant below an electric field of $2 \mathrm{MV} / \mathrm{cm}(3 \mathrm{MV} / \mathrm{cm})$

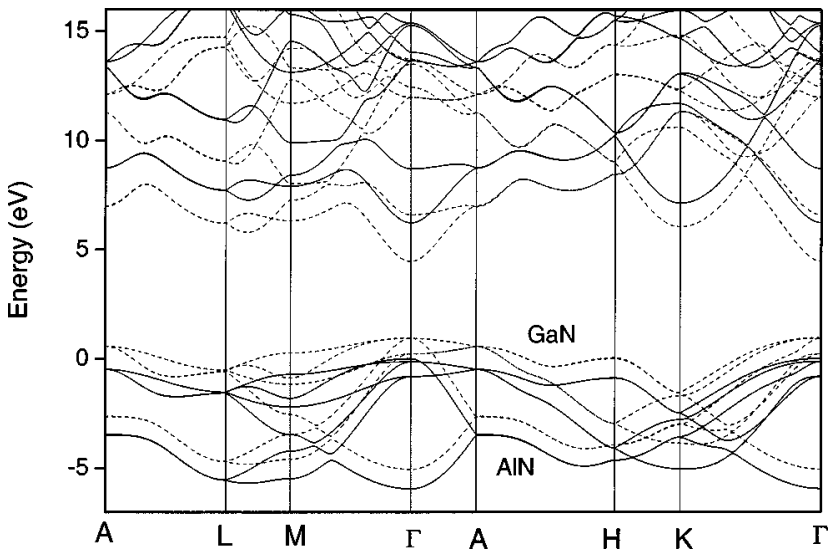

FIG. 1. (Color online) Band structure of GaN (dashed) and AlN (solid) computed using the empirical pseudopotential method. The valence-band offset between GaN and AlN is determined by 65/35 ratio of partitioning of the total band-gap difference, which is incorporated here solely to resolve these curves.

for GaN (AlN). It needs to be mentioned that we use the actual density of states, rather than the valley-based nonparabolic band approximation, in calculating the scattering rates. ${ }^{20}$ This becomes particularly important in the accurate characterization of the ODP mechanism. Akis et al. have recently verified in the zinc-blende phase of GaN that a oneparameter (deformation potential) fitting based on the density-of-states profile leads to a perfect agreement with the first-principles treatment of nonpolar scattering rate. ${ }^{21} \mathrm{We}$ use their fitted deformation potential value (1.32 $\times 10^{9} \mathrm{eV} / \mathrm{cm}$ ) for both $\mathrm{GaN}$ and $\mathrm{AlN}$ and associate this to an overall nonpolar optical phonon scattering, representing all optical phonon branches and polarizations. Figures 1 and 2 show our band structure and the corresponding density of states; for clarity, two set of bands are displaced by an amount equal to $65 / 35$ partitioning of the band-gap offset between the conduction and valence bands of $\mathrm{GaN}$ and $\mathrm{AlN}$

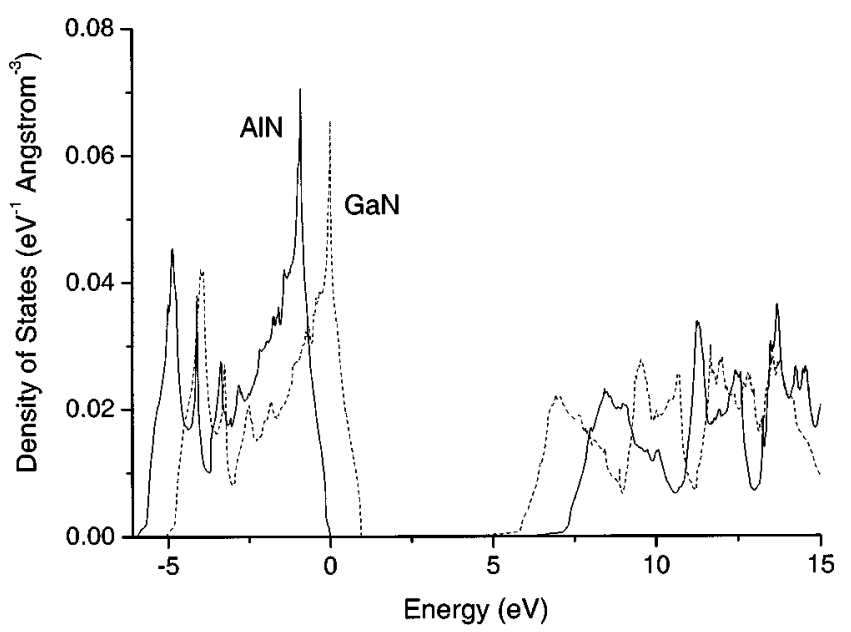

FIG. 2. (Color online) Density of states per spin, per unit volume for $\mathrm{GaN}$ (dashed) and AlN (solid). The valence-band offset between $\mathrm{GaN}$ and $\mathrm{AlN}$ is determined by 65/35 ratio of partitioning of the total band-gap difference, which is incorporated here solely to resolve these curves. 


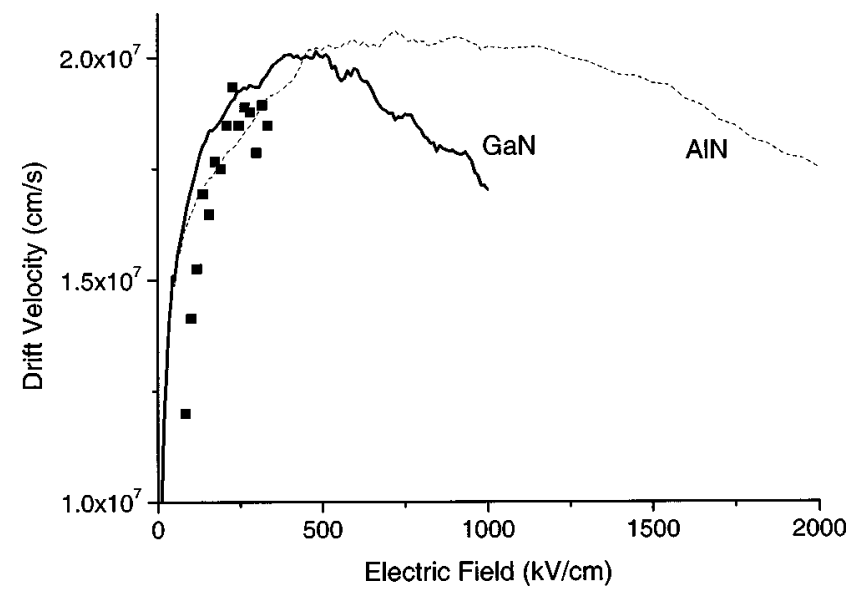

FIG. 3. (Color online) Steady-state velocity vs field for GaN (solid) and AlN (dashed) obtained by the EMC simulation; square symbols represent measurement values (Ref. 23) for GaN.

as if a heterojunction were to be formed. ${ }^{22}$ As a check for our EMC methodology, in Fig. 3 we display the steady-state velocity-field characteristics for GaN and AlN, comparing with the available experimental results in the case of $\mathrm{GaN}^{23}$ Note that in the low-field regime EMC results lead to much higher mobility compared to measured values, which is expected, as we do not include scattering mechanisms that govern the carrier dynamics in this regime such as the dislocation and piezoacoutic scatterings. We refer to Ref. 24 for a comprehensive theoretical consideration of mobility in GaN. For the high-field behavior, we obtain a better agreement with experiment in terms of the value of the peak velocity compared to previous theoretical estimations, ${ }^{25,26}$ however, the corresponding electric-field value seems to be somewhat higher in our case.

For a spatially uniform system, the time evolution of the (ensemble) average energy and momentum of the carriers can be described by one-dimensional balance equations ${ }^{27}$ as

$$
\begin{gathered}
\frac{d\langle E\rangle}{d t}=q F\left\langle v_{z}\right\rangle-\frac{\langle E\rangle-E_{0}}{\tau_{e}}, \\
\frac{d\left\langle p_{z}\right\rangle}{d t}=q F-\frac{\left\langle p_{z}\right\rangle}{\tau_{m}},
\end{gathered}
$$

using the energy and momentum relaxation times, $\tau_{e}$ and $\tau_{m}$, respectively. Here, the applied electric field $F$ is assumed to be along the $z$ axis, $q$ is the electronic charge, $\langle v\rangle$, $\langle E\rangle$, and $\langle p\rangle$ are the ensemble-average electron velocity, energy (with respect to conduction-band minimum), and momentum, respectively; these quantities are readily available from EMC simulations. The zero-field (i.e., thermal) mean energy is $E_{0}$, and that of momentum is zero. The energy and momentum relaxation times in Eqs. (1) and (2) are the macroscopic parameters which depend on the average electron energy. If one is interested in calculating the ensembleaverage electron energy and (drift) velocity using Eqs. (1) and (2), then of course these parameters should be specified. The main problem in this case is that this will require the

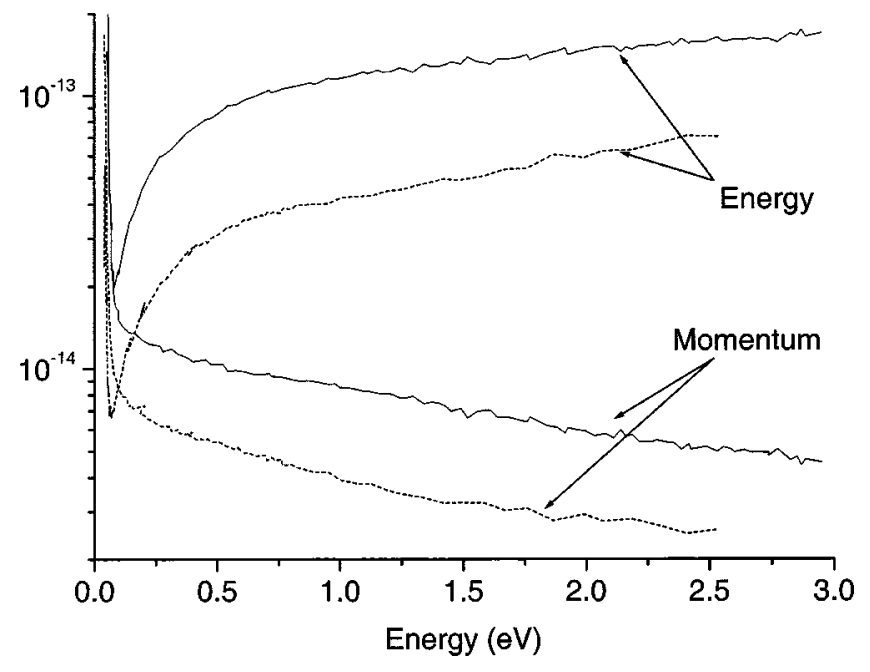

FIG. 4. (Color online) Energy and momentum relaxation times (in s) for GaN (solid) and AlN (dashed) calculated using EMC.

solution of the microscopic electron kinetic equation which includes all actual microscopic scattering mechanisms. In this case, when solution of the kinetic equation is obtained, Eqs. (1) and (2) would provide little additional information. On the other hand, Eqs. (1) and (2) may serve as a definition of the macroscopic energy and momentum relaxation times provided that one knows the average energy and momentum (velocity). This approach, which was suggested by Shur (see Ref. 27 and references therein), is usually employed in combination with the EMC simulation. We follow this approach here as well. Invoking the steady-state conditions $\left(d\left\langle p_{z}\right\rangle / d t \rightarrow 0\right.$ and $\left.d\langle E\rangle / d t \rightarrow 0\right)$ yields the relaxation rates as

$$
\begin{gathered}
\tau_{e}=\frac{\langle E\rangle-E_{0}}{q F\left\langle v_{z}\right\rangle}, \\
\tau_{m}=\frac{\left\langle p_{z}\right\rangle}{q F} .
\end{gathered}
$$

In passing, it can be pointed out that even though we include the impact ionization in the scattering processes, this mechanism is mainly connected with the tail of the distribution function and does not directly affect the ensemble-average quantities considered in this section.

In Fig. 4 we show the momentum and energy relaxation times for GaN and AlN, as a function of mean carrier energy which is compiled from a large number of EMC simulations, each at a higher electric value. One convenience of the EMC approach is that the effect of each scattering mechanism can easily be singled out by "switching" it off. By this means we identify that POP scattering dominates up to an electric-field value of about $300 \mathrm{kV} / \mathrm{cm}(600 \mathrm{kV} / \mathrm{cm})$ for $\mathrm{GaN}(\mathrm{AlN})$; above this value the ODP mechanism takes over. At these transition fields, the steady-state velocity attains its peak value (see Fig. 3) above which the higher valleys begin to dominate the transport characteristics, giving rise to negative differential mobility. Finally, we observe that the energy relaxation times for both GaN and AlN display an opposite 
trend with respect to the momentum relaxation times as a function of energy; similar behavior was also obtained recently by Herbert et al. ${ }^{28}$ which is a hallmark of the hotelectron phenomena.

\section{FULL-BAND FIRST-PRINCIPLES ANALYSIS}

The EMC treatment in the preceding section, based on the semiclassical Boltzmann equation essentially characterizes the incoherent carrier dynamics. Even more intriguing is the coherent dynamics ${ }^{29,30}$ which has become an experimental possibility with the advent of ultrafast optical probing techniques. ${ }^{31}$ In these processes, carriers are coherently excited to an energetic quantum state $\mathbf{k}$ and it is the outscattering characteristics of this $\mathbf{k}$ state that is predominantly important for the subsequent carrier dynamics. With this motivation, in this section we consider the relevant relaxation rates for the dominant POP and ODP scattering mechanisms at each $\mathbf{k}$ state along high-symmetry lines. The computational approach chosen for an efficient execution of these expressions is also included, nevertheless, for an unabridged account we refer the reader to our previous work. ${ }^{9}$ As a cautionary remark, this approach as well as EMC make use of Fermi's golden rule. However, on very short time scales, even the description of scattering processes in terms of rates obtained from Fermi's golden rule becomes questionable and a quantum-kinetic framework may be more appropriate which is, on the other hand, much more involved. ${ }^{32,33}$

\section{A. Scattering, momentum and energy relaxation expressions}

Starting from Fermi's golden rule, the total out-scattering rate from an initial state due to POP emission, considering only normal processes, is given by

$$
\begin{aligned}
W_{j, m}(\mathbf{k}) & =\sum_{m^{\prime}} \int_{1 \mathrm{st} \mathrm{BZ}} \hat{W}_{j, m^{\prime}}\left(\mathbf{k}^{\prime}\right) \delta\left[E_{m^{\prime}}\left(\mathbf{k}^{\prime}\right)-E_{m}(\mathbf{k})+\hbar \omega_{j, \mathbf{q}}\right] \\
& =\sum_{m^{\prime}} \int_{S} d S \frac{\hat{W}_{j, m^{\prime}}\left(\mathbf{k}^{\prime}\right)}{\left|\boldsymbol{\nabla} E_{m^{\prime}}\left(\mathbf{k}^{\prime}\right)\right|}
\end{aligned}
$$

where

$$
\hat{W}_{j, m^{\prime}}\left(\mathbf{k}^{\prime}\right)=\frac{2 \pi}{\hbar} \frac{V}{(2 \pi)^{3}} \Delta_{m^{\prime}, m}\left(\mathbf{k}^{\prime}, \mathbf{k}\right)\left|C_{j}(\mathbf{q})\right|^{2}\left(n_{j, \mathbf{q}}+1\right)
$$

The labels $m$, k represent the initial-state electron band index and wave vector, respectively; primed indices correspond to the final-state, after the out-scattering event. The cellperiodic overlap parameter is given by

$$
\Delta_{m^{\prime}, m}\left(\mathbf{k}^{\prime}, \mathbf{k}\right)=\left|\frac{1}{\Omega} \int_{\Omega} u_{m^{\prime}, \mathbf{k}^{\prime}}^{*}(\mathbf{r}) u_{m, \mathbf{k}}(\mathbf{r}) d^{3} r\right|^{2}
$$

where $u_{m, \mathbf{k}}(\mathbf{r})$ is the cell-periodic part of the Bloch function and $\Omega$ is the volume of the primitive cell. The integration is over the surface $S$ described by the energy-conservation equation for the one-phonon-emission process, $E_{m^{\prime}}\left(\mathbf{k}^{\prime}\right)$
$=E_{m}(\mathbf{k})-\hbar \omega_{j, \mathbf{q}}$. Here, $\hbar \omega_{j, \mathbf{q}}$ is the phonon energy corresponding to wave vector $\mathbf{q}=\mathbf{k}^{\prime}-\mathbf{k}$ mapped to first Brillouin zone (BZ) and $j$ is the polar optical phonon branch index. For the wurtzite structure, which is the stable crystal phase for both GaN and AlN, the polar phonon branches are longitudinal-optical- (LO) like and transverse-optical- (TO) like, ${ }^{9}$ whereas in the zinc-blende phase it is a pure LO mode. These electron-POP coupling coefficients $C_{j}(\mathbf{q})$ have already been given, including the associated material parameters for $\mathrm{GaN}$ and $\mathrm{AlN},{ }^{9,11}$ and will not be repeated here. However, note that in these materials the optical phonon energy is about four times the thermal energy at room temperature. For this reason, phonon occupation probability $n_{j}(\mathbf{q})$ becomes negligible up to room temperature, so that we can essentially set $n_{j}(\mathbf{q}) \equiv 0$. This is also the reason why we ignore the POP absorption processes in our consideration.

The integrand of the scattering rate expression in Eq. (5) needs to be weighted by

$$
1-\frac{k^{\prime}}{k} \cos \alpha
$$

and

$$
1-\frac{E_{m^{\prime}}\left(\mathbf{k}^{\prime}\right)}{E_{m}(\mathbf{k})} \stackrel{\text { POP emission }}{\rightarrow} \frac{\hbar \omega_{j}}{E_{m}(\mathbf{k})},
$$

in the case of momentum and energy relaxation rates, respectively, with $\alpha$ corresponding to the angle between $\mathbf{k}$ and $\mathbf{k}^{\prime}$.

A requirement of a first-principles scattering rate computation is the efficient evaluation of BZ integrals, like Eq. (5). Such tools were developed several decades ago, among which we prefer the Lehmann-Taut technique. ${ }^{34}$ In the implementation we divide the irreducible wedge of the first BZ into a fine tetrahedra, and store the band energies and the cell-periodic overlap parameters at the nodes of these tetrahedra. Other details of our technique can be found in Refs. 9,11 .

The scattering rate for the (nonpolar) ODP scattering at zero temperature is given by

$$
W(E)=\pi D_{\mathrm{LO}}^{2} \frac{N\left(E-\hbar \omega_{\mathrm{LO}}\right)}{\rho \omega_{\mathrm{LO}}}
$$

where $\rho$ is the mass density and $D_{\mathrm{LO}}$ is the deformation potential constant which is taken as $1.32 \times 10^{9} \mathrm{eV} / \mathrm{cm}$ for both $\mathrm{GaN}$ and $\mathrm{AlN}$, as mentioned in the preceding section. The corresponding phonon energy, $\hbar \omega_{\mathrm{LO}}$ is taken as the zone-boundary TO phonon energy, is $65.8 \mathrm{meV}(80.9 \mathrm{meV})$ for GaN (AlN). Finally, $N(\cdots)$ is the density of states per spin, per unit volume, shown in Fig. 2.

\section{B. Results}

We trace the POP and ODP scattering rates of the conduction-band electrons, starting from the conductionband minimum at the $\Gamma$ point, along high-symmetry lines: $\Gamma-M, \Gamma-K, \Gamma-A$ or $\Gamma-U_{3, \min }$. Here, the importance of the point $U_{3, \min }$ which is located on the line joining points $M$ to $L$ is that, for $\mathrm{GaN}$, it corresponds to the lowest satellite valley 

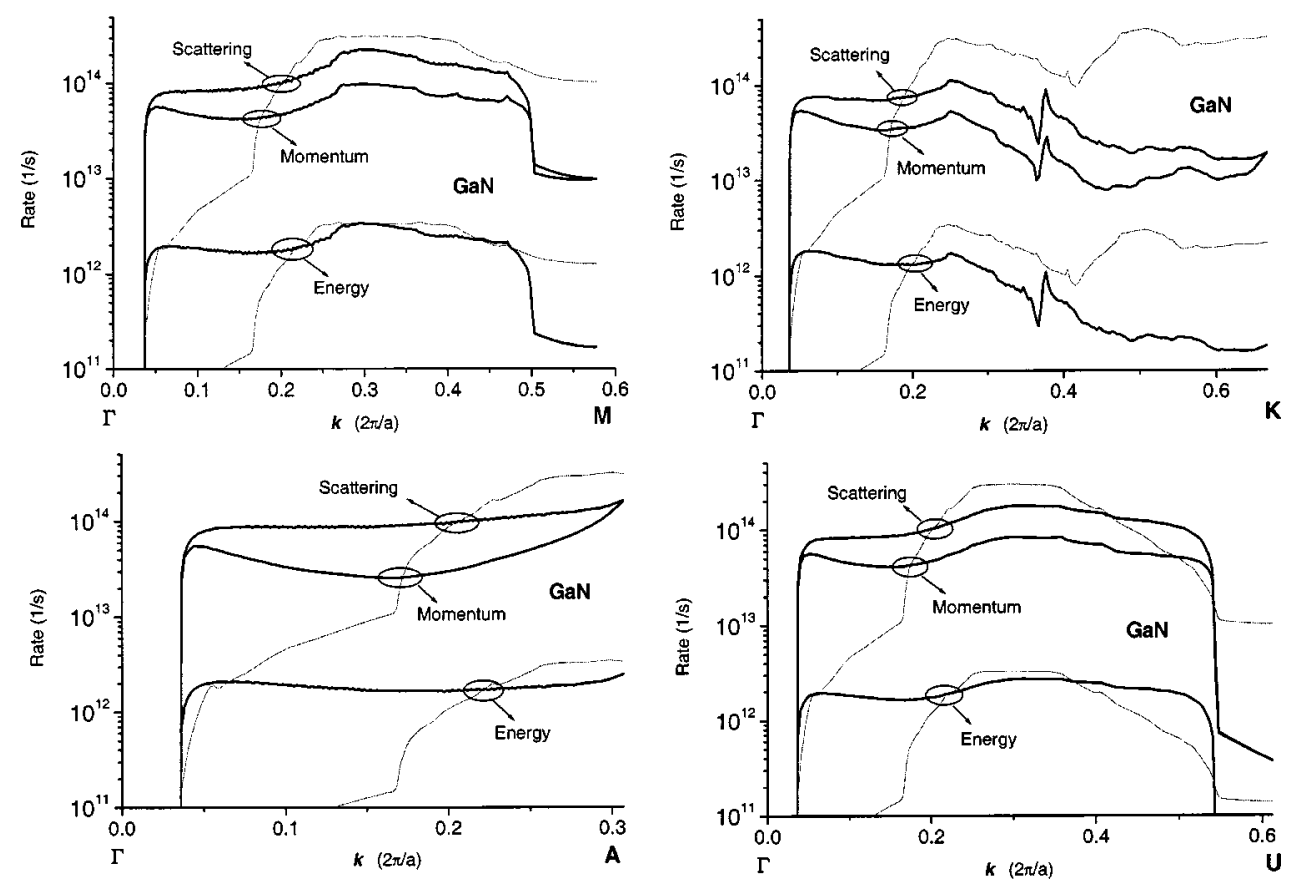

FIG. 5. (Color online) Scattering, momentum and energy relaxation rates for GaN due to POP (thick lines) and ODP (thin red lines) mechanisms.

in the conduction band. The results are shown in Figs. 5 and 6 for GaN and AlN, respectively; only the LO-like POP branch is considered, as the TO-like scattering rate was found to be two orders of magnitude smaller. ${ }^{9}$ The ODP rates simply follow the density of states, therefore, the POP mechanism deserves more description.

The satellite valleys play major role in the quantitative value of the POP scattering rates: As soon as scattering to other satellite valleys becomes energetically possible, the scattering rate significantly increases from its band-edge value, and towards $U$ and $M$ valleys it suddenly drops when the intravalley scattering is no longer available. Momentum relaxation rate, starting from the band edge, begins to decrease significantly from that of the scattering rate, as predicted by simple parabolic band considerations ${ }^{7,8}$ which is due to forward-scattering nature of the intravalley POP mechanism. However, towards the BZ boundary, the intervalley scattering becomes the only viable channel which has an isotropic character. Therefore, the $\cos \alpha$ term in Eq. (9) of the momentum relaxation rate averages out to zero, removing the discrepancy with the ordinary scattering rate, which is also confirmed by the results in Figs. 5 and 6. Finally, the
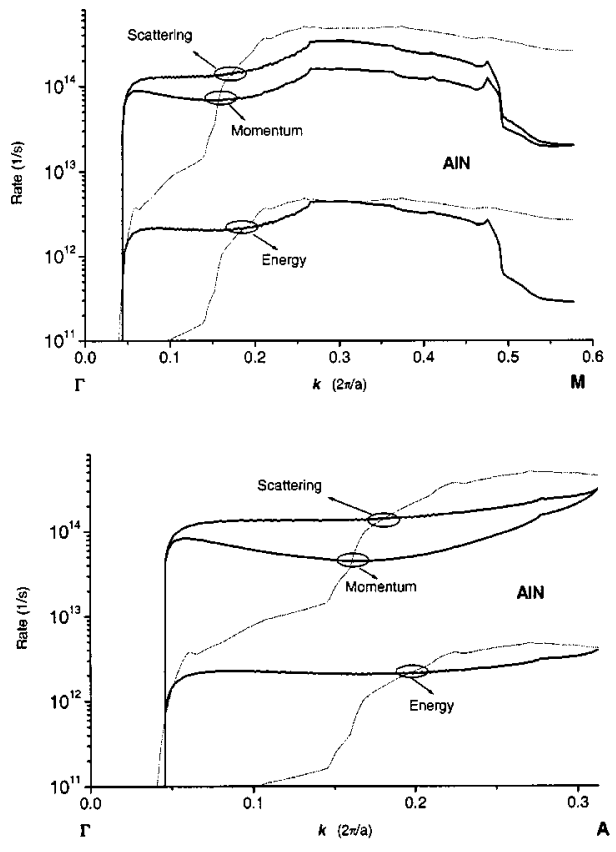
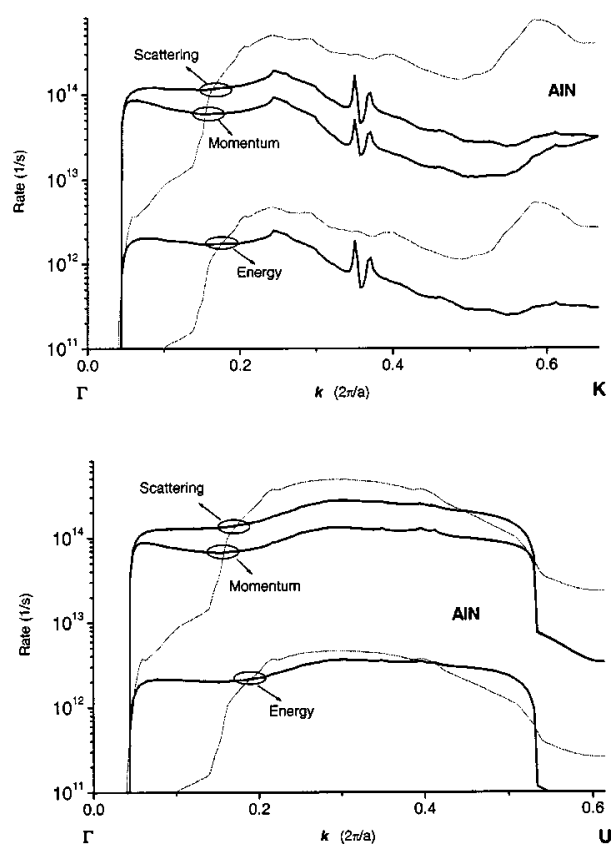

FIG. 6. (Color online) Scattering, momentum and energy relaxation rates for AlN due to POP (thick lines) and ODP (thin red lines) mechanisms. 
energy relaxation rate is about two orders of magnitude lower, due to minute value of the POP energy compared to those of highly energetic electrons assumed along these highsymmetry directions [see, Eq. (10)], as was mentioned earlier. Moreover, both GaN and AlN display very similar behavior, with AIN rates being about 50\% higher than those of $\mathrm{GaN}$ as an outcome of its higher ionicity.

Unlike the POP case, ODP mechanism has an isotropic scattering pattern, rendering momentum relaxation rate equal to the total out-scattering rate. For both $\mathrm{GaN}$ and AlN roughly above an energy of $1.5 \mathrm{eV}$ with respect to the conduction-band minimum the ODP rate exceeds the POP value which is due to sudden rise of the associated density of states (see Fig. 2). As another noteworthy point, the momentum relaxation rates obtained using EMC analysis shown in Fig. 4 are significantly higher than those in Figs. 5 and 6. The main reason for this is that EMC results are based on ensemble-average energies where a substantial amount of carriers already occupy higher conduction bands being exposed to much higher scattering, whereas those in Figs. 5 and 6 trace the lowest conduction band.

\section{Krömer-Esaki-Tsu negative differential mobility}

Based on an idea that goes back to Krömer, ${ }^{12}$ there exists the possibility of a negative differential mobility driven by the band structure's negative effective mass part beyond the inflection point of its dispersion curve. The dependence of the velocity on the applied electric field can easily be estimated by the simple approach used by Esaki and Tsu for superlattices. ${ }^{13}$ Hence, the average velocity, assuming a constant scattering time $\tau$, is given by

$$
v_{d}=e F \hbar^{-2} \int_{0}^{\infty} \frac{\partial^{2} E}{\partial k^{2}} e^{-t / \tau} d t
$$

where $\partial^{2} E / \partial k^{2}$ is the curvature of the energy band diagram along the applied field's direction $\mathbf{F}$, sampled at the $\mathbf{k}$ point, $k(t)=e F t / \hbar$, for an electron originating from the $\Gamma$ point. In our previous analysis, ${ }^{9,11}$ for the characteristic scattering time $\tau$ in this equation, we used the value given by the minimum POP scattering time along each direction. More realistically, in this regime, the character of the electron motion in the momentum space is rather intermediate between the diffusionlike and the ballisticlike motion, which are characterized by the momentum relaxation time and the scattering time, respectively. Therefore, we would like to supplement this estimate with those based on the POP momentum relaxation rate and the ODP scattering rate. Figure 7 shows the corresponding high-field velocity behaviors for both $\mathrm{GaN}$ and AlN along the $\Gamma-M$ direction. If only POP mechanism were operational, we would expect the resultant curve to lie in between the $\operatorname{POP}(s)$ and $\operatorname{POP}(m)$ curves, however, the presence of ODP scattering shifts this interesting effect to much higher fields where these materials are likely to breakdown beforehand. Finally, compared to the EMC analysis in Fig. 3 referring to incoherent dynamics which is fine up to a few $\mathrm{MV} / \mathrm{cm}$ values, at even higher fields ballistic and semiballistic (lucky drift) processes ${ }^{7}$ become important and it is these
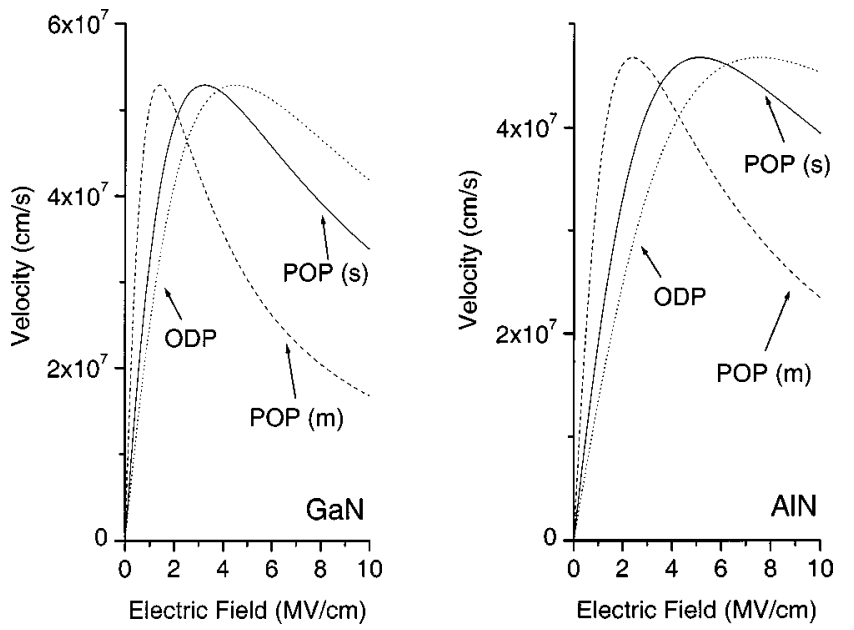

FIG. 7. High-field velocity behavior along $\Gamma-M$ direction for both GaN and AlN. For the scattering time terminating the ballistic motion, three cases are illustrated: POP scattering time (solid), POP momentum relaxation time (dashed), and ODP scattering/ momentum relaxation time (dotted).

that not only govern the impact ionization but may also introduce the negative differential mobility depicted in Fig. 7, which introduces a quite different physical situation. However, a more rigorous treatment based on a quantum-kinetic formulation ${ }^{32,33}$ should critically examine these findings.

\section{CONCLUSIONS}

A comprehensive account of scattering, momentum and energy relaxation rates in $\mathrm{GaN}$ and $\mathrm{AlN}$ is given based on EMC and first-principles full-band approaches, for assessing the incoherent and coherent electron dynamics, respectively. Disparities among these relaxation rates govern the character of the electronic motion in the momentum space, causing dissipative, diffusive, or ballistic behaviors. Our EMC analysis indicates that POP mechanism dominates up to the fields where peak steady-state velocity is attained, and the ODP process takes over afterwards. Our full-band first-principles approach, on the other hand, characterizes these rates at each $\mathbf{k}$ state along a high-symmetry line which is more useful in characterizing coherent dynamics. In this case as well, the POP mechanism reigns in the low-energy region, roughly up to a value of $1.5 \mathrm{eV}$, above which the ODP process dominates due to strong increase in the associated density of states. For the ODP case, both scattering and momentum relaxation rates coincide due to its isotropic scattering pattern, whereas for POP, especially in the region where it is dominant, momentum relaxation rate is significantly lower than the scattering rate due to its forward-scattering behavior. This implies that under a high electric field even though an electron makes several collisions on its way to the zone boundary, its progress is not as impeded as the scattering rate would suggest. This difference between the momentum relaxation time and the scattering time is of crucial importance for the evaluation of the feasibility of both the full-band electron dynamics (Krömer-Esaki-Tsu regime) in bulk semi- 
conductors, and the overshoot regime in GaN-based HEMT's with the gate length of a few tens of nanometers. Finally, given the concerns on the validity of Fermi's golden rule in ultrashort time scales, a quantum-kinetic approach ${ }^{32,33}$ may also be very useful as a follow up of this work.

\section{ACKNOWLEDGMENTS}

C.B. would like to thank The Scientific and Technical Research Council of Turkey (TÜBITAK) and Bilkent University for their support.
*Electronic address: bulutay@fen.bilkent.edu.tr

†Electronic address: bkr@essex.ac.uk

†Electronic address: nick.zakhleniuk@bookham.com

${ }^{1}$ L.F. Eastman, Phys. Status Solidi A 176, 175 (1999).

${ }^{2}$ M.S. Shur, Solid-State Electron. 42, 2131 (1998).

${ }^{3}$ E. Muñoz, E. Monroy, J.L. Pau, F. Calle, F. Omnès, and P. Gibart, J. Phys.: Condens. Matter 13, 7115 (2001).

${ }^{4}$ N.M. Stanton, A.J. Kent, A.V. Akimov, P. Hawker, T.S. Cheng, and C.T. Foxon, J. Appl. Phys. 89, 973 (2001).

${ }^{5}$ N. Balkan, M.C. Arikan, S. Gokden, V. Tilak, B. Schaff, and R.J. Shealy, J. Phys.: Condens. Matter 14, 3457 (2002); A. Matulionis, J. Liberis, L. Ardaravičius, M. Ramonas, I. Matulioniene, and J. Smart, Semicond. Sci. Technol. 17, L9 (2002).

${ }^{6} \mathrm{M}$. Farahmand and K.F. Brennan, IEEE Trans. Electron Devices 47, 493 (2000).

${ }^{7}$ B.K. Ridley, Quantum Processes in Semiconductors, 4th ed. (Oxford University Press, Oxford, 1999).

${ }^{8}$ M. Lundstrom, Fundamentals of Carrier Transport, 2nd ed. (Cambridge University, Cambridge, 2000).

${ }^{9}$ C. Bulutay, B.K. Ridley, and N.A. Zakhleniuk, Phys. Rev. B 62, 15754 (2000).

${ }^{10}$ C. Bulutay, B.K. Ridley, and N.A. Zakhleniuk, Appl. Phys. Lett. 77, 2707 (2000).

${ }^{11}$ C. Bulutay, B.K. Ridley, and N.A. Zakhleniuk, Physica B 314, 63 (2002).

${ }^{12}$ H. Krömer, Phys. Rev. 109, 1856 (1958).

${ }^{13}$ L. Esaki and R. Tsu, IBM J. Res. Dev. 14, 61 (1970).

${ }^{14}$ D. Vasileska and S.M. Goodnick, Mater. Sci. Eng., R. 38, 181 (2002).

${ }^{15}$ M.A. Osman and D.K. Ferry, Phys. Rev. B 36, 6018 (1987).

${ }^{16}$ P. Lugli and D.K. Ferry, IEEE Trans. Electron Devices 32, 2431 (1985)

${ }^{17}$ H.M. van Driel, Phys. Rev. B 19, 5928 (1979); W. Pötz and P. Kocevar, Phys. Rev. B 28, 7040 (1983); P. Kocevar, Physica B \& C 134, 155 (1985).
${ }^{18}$ P. Lugli, P. Bordone, L. Reggiani, M. Rieger, P. Kocevar, and S.M. Goodnick, Phys. Rev. B 39, 7852 (1989).

${ }^{19}$ C. Bulutay, Semicond. Sci. Technol. 17, L59 (2002).

${ }^{20}$ M.V. Fischetti and J.M. Higman, Monte Carlo Device Simulation: Full Band and Beyond, edited by K. Hess (Kluwer Dordrecht, 1991).

${ }^{21}$ R. Akis, M. Dür, and S.M. Goodnick, Physica B 314, 42 (2002).

${ }^{22}$ D.R. Hang, C.H. Chen, Y.F. Chen, H.X. Jiang, and J.Y. Lin, J. Appl. Phys. 90, 1887 (2001).

${ }^{23}$ M. Wraback, H. Shen, J.C. Carrano, T. Li, J.C. Campbell, M.J. Schurman, and I.T. Ferguson, Appl. Phys. Lett. 76, 1155 (2000).

${ }^{24}$ S. Dhar, and S. Ghosh, J. Appl. Phys. 86, 2668 (1999).

${ }^{25}$ B.E. Foutz, S.K. O'Leary, M.S. Shur, and L.F. Eastman, J. Appl. Phys. 85, 7727 (1999).

${ }^{26}$ J.D. Albrecht, R.P. Wang, P.P. Ruden, M. Farahmand, and K.F. Brennan, J. Appl. Phys. 83, 4777 (1998); J.D. Albrecht, R.P. Wang, P.P. Ruden, M. Farahmand, and K.F. Brennan, ibid. 83, 1446 (1998).

${ }^{27}$ M. Shur, GaAs Devices and Circuits (Plenum Press, New York, 1987), Chap. 2.

${ }^{28}$ D.C. Herbert, M.J. Uren, B.T. Hughes, D.G. Hayes, J.C.H. Birbeck, R. Balmer, T. Martin, G.C. Crow, R.A. Abram, M. Walmsley, R.A. Davies, R.H. Wallis, W.A. Phillips, and S. Jones, J. Phys.: Condens. Matter 14, 3479 (2002).

${ }^{29}$ Coherent Control in Atoms, Molecules, and Semiconductors, edited by W. Pötz and W.A. Schroeder (Kluwer, Dordrecht, 1999).

${ }^{30}$ F. Rossi, Semicond. Sci. Technol. 13, 147 (1998); F. Rossi and T. Kuhn, Rev. Mod. Phys. 74, 895 (2002).

${ }^{31}$ J. Shah, Ultrafast Spectroscopy of Semiconductors and Semiconductor Nanostructures (Springer, Berlin, 1999).

${ }^{32}$ H. Haug and A.-P. Jauho, Quantum Kinetics in Transport and Optics of Semiconductors (Springer, Berlin, 1996).

${ }^{33}$ M. Bonitz, Quantum Kinetic Theory (Teubner, Stuttgart, 1998).

${ }^{34}$ G. Lehmann and M. Taut, Phys. Status Solidi B 54, 469 (1972). 